\title{
Comparison of enzymic with cineangiocardiographic estimations of myocardial infarct size ${ }^{\star}$
}

\author{
N L SAMMEL, J G STUCKEY, $†$ P W T BRANDT, R M NORRIS + \\ From Green Lane Hospital, Auckland 3, New Zealand
}

SUMMARY Comparisons were made between enzymic indices of myocardial infarct size (total creatine kinase appearance and peak enzyme activity) measured during the acute stage of a first myocardial infarct in 32 male patients, and analysis of contraction abnormalities in biplane left ventricular cineangiocardiograms performed one month later. The cineangiocardiograms were analysed independently by two radiologists, each using two different methods for quantification of subjectively classified abnormalities of left ventricular wall motion. A very strong correlation was found between the two enzymic indices of infarct size and somewhat weaker correlations between assessment of contractility abnormalities made by the two radiologists using the same method, or by the same radiologist using the two different methods. Comparisons between enzymic and angiocardiographic indices for all infarcts showed correlation coefficients $(r)$ within the range of 0.53 to 0.72 . With all comparisons of enzymic with radiological indices $r$ values were higher for anterior infarcts than for inferior infarcts, and there was a tendency for higher enzyme levels for a given degree of left ventricular damage in inferior than in anterior infarction. This may be the result of variable degrees of right ventricular damage in inferior infarction.

Mortality and morbidity after myocardial infarction are largely related to the extent of myocardial damage, ${ }^{1}$ and in recent years have prompted the evaluation of methods to reduce infarct size in patients. $^{2-4}$ Estimation of cumulative creatine kinase appearance into the blood from an infarcted region (integral $\mathrm{CK}$ ) is a common method for the assessment of myocardial damage and evaluation of therapeutic interventions. ${ }^{256}$

Most authors have reported a good correlation between myocardial damage as estimated by serum $\mathrm{CK}$ and other means, such as necropsy findings, biplane cineangiocardiography, ${ }^{8}$ and myocardial CK depletion in experimental myocardial infarction. ${ }^{910}$ Criticism of integral CK, however, continues both regarding the assumptions made in its calculation ${ }^{11} 12$ as well as its correlation with histological infarction size in experimental animals. ${ }^{13}$

Our study attempts to shed further light on the controversy by comparing infarct size measured by total CK appearance with peak CK activity, as well

\footnotetext{
* This work was supported by the Medical Research Council and The National Heart Foundation of New Zealand.

† Present address: Department of Radiology, Royal Melbourne Hospital, Victoria 3050, Australia.

$¥$ Present address: Department of Cardiology, The Radcliffe

Infirmary, Oxford OX2 6HE, England.

Received for publication 8 October 1979
}

as with biplane cineangiocardiographic assessments of myocardial damage using subjective analysis of both the ventriculograms and coronary arteriograms.

\section{Patients and methods}

Thirty-eight consecutive male patients under 60 years of age seen within 12 hours of the onset of symptoms of an uncomplicated first myocardial infarct were entered in the study. Seven patients were subsequently excluded after analysis of left ventriculography and coronary arteriography In two, the left ventriculogram was technically unsatisfactory, and in five previous myocardial infarction could not be excluded in the presence of either two separate areas of left ventricular dysfunction (two patients) or a single abnormal region supplied by more than one occluded vessel (three patients). Our findings refer to the remaining 31 patients. Management in the coronary care unit was of a routine nature with the administration of antiarrhythmic drugs (usually lignocaine), frusemide, and analgesics (usually papaveretum) when necessary. Some patients were included in the continuation of a clinical trial of propranolol in acute myocardial infarction. ${ }^{6}$ No drugs were given intramuscularly, and patients undergoing cardio- 
version were excluded from the study. Blood for $\mathrm{CK}$ sampling was withdrawn from an intravenous cannula inserted at the time of admission. Sampling was performed four-hourly for 72 hours or until CK, as measured by the Rosalki procedure at $30^{\circ} \mathrm{C}^{14}$ returned close to the normal range. Cumulative $\mathrm{CK}$ appearance was calculated by our previously described modification ${ }^{15}$ of the method of Shell and Sobel, ${ }^{1}$ and the peak level of CK activity was also noted for each patient.

Left ventriculography and selective coronary arteriography were offered to patients at one month after admission to hospital as part of a wider study of prognosis and the place of coronary surgery after a first infarction. Treadmill stress testing was performed routinely before angiography.

Left ventriculography and selective coronary arteriography were performed by the Judkins procedure, ${ }^{16}$ and all left ventriculograms were recorded biplane on $35 \mathrm{~mm}$ cine film. The projections used were $35^{\circ}$ right anterior oblique (RAO) and 25 to $35^{\circ}$ hemiaxial $55^{\circ}$ left anterior oblique (LAO). Two methods of estimating infarct size from the cine studies were used, each method being used independently by two radiologists (JS and PB). For the first, termed the "contractility" method, the left ventricle was arbitrarily divided into nine segments (Fig. 1). Contractility of each segment
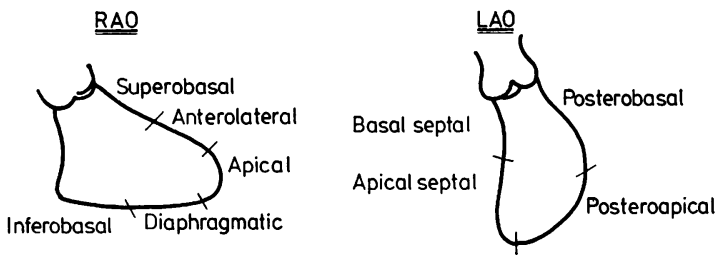

Fig. 1 Left ventricular segments. "Contractility" method. The diagram on the left shows the five segments in a $35^{\circ}$ right anterior oblique projection ( $\left.R A O\right)$, and the diagram on the right shows the four segments in a $55^{\circ}$ left anterior oblique projection with a $30^{\circ}$ cranial axial tilt (LAO).

was assessed subjectively and given a "score" as normal (no points), hypokinetic (one point), akinetic (two points), or dyskinetic (three points). As the septum contains about half the left ventricular muscle, ${ }^{17}$ yet is profiled in only two of the nine segments, the septal segments were given a multiplication factor of three. Theoretical maximum score (given the impossible situation of all segments being dyskinetic) would be 39. Fig. 2 shows the score for a patient who had akinesia of the anterolateral and apical segments, dyskinesia of the apical septal segment, and normal contractility in the remainder of the profiled segments.

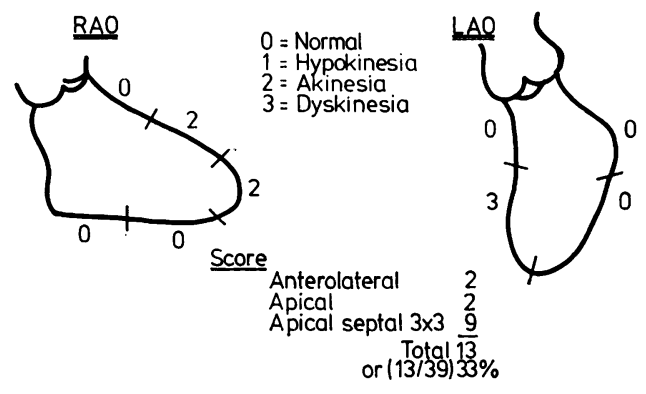

Fig. 2 Angiocardiographic score- "contractility" method. This is an example of the calculation of the score with this method in a patient with akinesia of the anterolateral and apical segments and dyskinesia of the apical septum.

The second radiological method for estimation of infarct size was termed the "muscle loss" method. This method was based on the contractile behaviour of the myocardium assessed not only by the appearance of the cavity margins in the biplane ventriculograms, but also by observing the motion of arteries in the septum and on the free wall in the coronary arteriograms. In addition, allowance was made for wall thinning and loss of trabeculation where this could be identified. This method evolved from the style of coronary arteriographic reporting used at Green Lane Hospital ${ }^{18}$ where the left ventricular myocardium is divided into defined regions and scored according to the estimated

Table 1 "Muscle loss" method; multiplication factors for severity

\begin{tabular}{ll}
\hline Angiographic interpretation & Severity \\
\hline Normal & 0 \\
Hypokinetic & 1 \\
Akinetic without muscle loss & 2 \\
Akinetic or dyskinetic with partial muscle loss & 3 \\
Akinetic or dyskinetic with total muscle losst & 4
\end{tabular}

* Partial wall thinning with some trabeculation visible.

+ Thin scar with total loss of trabeculations.

Table 2 "Muscle loss" assessment of patient with inferior infarction

\begin{tabular}{llll}
\hline Region & Units & Severity & Score \\
\hline Anterior septum & 5 & 0 & 0 \\
Posterior septum & 2 & 2 & 4 \\
Diagonal & 2 & 0 & 0 \\
Obtuse margin & 2 & $\left\{\begin{array}{l}1 \\
0\end{array}\right.$ \\
Inferior LV wall & 1 & 3 & 9 \\
Total & 3 & - & $15(25 \%) \dagger$ \\
\hline
\end{tabular}

* See Brandt et al. ${ }^{18}$.

+ Expressed as a percentage of theoretical maximal score 60 (multiplication factor of 4 for 15 myocardial units). 
amount of myocardium affected as well as the severity of arterial obstructions. For the estimation of infarct size the severity of observed arterial narrowings was ignored, but the left ventricular myocardium was considered in terms of 15 myocardial units, seven in the septum (divided into anterior and posterior septal regions), two in the diagonal area, and three each on the obtuse margin and inferior surfaces. A subjective estimation of contractile state of each anatomical zone was made using all the information available in the cine studies and using a severity multiplication factor as in Table 1. A complete "muscle loss" assessment in shown in Table 2.

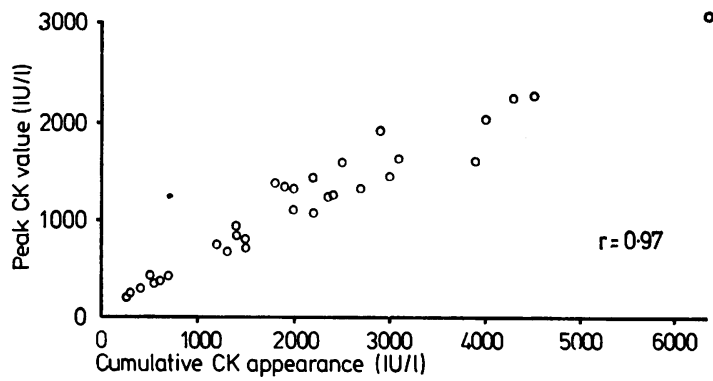

Fig. 3 Comparison of infarct size assessed by peak $C K$ value and cumulative $C K$ appearance. $A$ strong correlation is shown $(r=0.97)$.

Table 3 Comparability of ventriculographic assessments

\begin{tabular}{lll}
\hline Subject for comparison & & $\begin{array}{c}\text { Correlation } \\
\text { coefficient } \\
(r j\end{array}$ \\
\hline $\begin{array}{l}\text { Two ventriculographic } \\
\text { methods }\end{array}$ & JS & 0.91 \\
$\begin{array}{l}\text { ("contractility", } \\
\text { "muscle loss") }\end{array}$ & PB & 0.89 \\
$\begin{array}{l}\text { Assessment of two } \\
\text { radiologists } \\
\text { (JS, PB) }\end{array}$ & "Contractility" method & 0.85 \\
\hline
\end{tabular}

\section{Results}

Fig. 3 depicts the very close correlation $(r=0.97)$ between the two enzymic indices. Table 3 shows the slightly weaker correlations $(r=0.85$ to 0.91$)$ between the two angiographic methods (contractility and muscle loss) in the hands of the same radiologist, and the assessments of the two radiologists (JS and PB), using the same method. An example of this is shown in Fig. 4. Table 4 compares the enzymic with the ventriculographic indices and shows correlation coefficients for all infarcts ranging from $r=0.53$ to $r=0.72$. An example of the weaker correlation between enzymic and ventriculographic methods appears in Fig. 5.

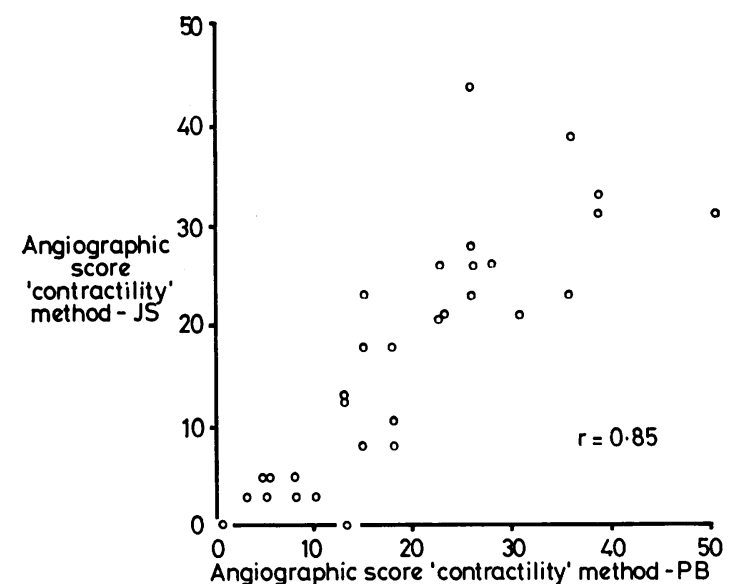

Fig. 4 Comparison of the two radiologists' angiocardiographic assessments of myocardial damage using the "contractility" method.

Correlations between enzymic and ventriculographic indices for patients with anterior infarcts ( $\mathrm{r}$ values ranging from 0.68 to 0.84 ) were better than those for inferior infarcts ( $r$ values from 0.51 to 0.71 ). There was also a tendency with inferior infarction for relatively more enzyme appearance for a given degree of left ventricular impairment. This tendency did not attain statistical significance, but was evident in all the enzymic/vertriculographic correlations (Fig. 5). Right ventricular infarction was considered likely in four patients with inferior myocardial infarction, who had occlusions of the right coronary artery proximal to all right ventricular branches. Supportive clinical features (right ventricular failure inappropriately severe for the degree of left ventricular failure) were seen in two of these patients. Exclusion of the four patients slightly improved the correlations for inferior infarcts ( $r$ values 0.62 to 0.77 ). In addition, right ventricular infarction may have occurred in other patients with inferior infarction and more distal lesions of the right coronary artery, but this could not be judged with any degree of accuracy.

Table 4 Comparison of enzymic and ventriculographic estimations of myocardial infarct size (correlation coefficients)

\begin{tabular}{|c|c|c|c|c|c|}
\hline & & \multicolumn{2}{|c|}{$\begin{array}{l}\text { "Contractility" } \\
\text { method }\end{array}$} & \multicolumn{2}{|c|}{$\begin{array}{l}\text { "Muscle loss" } \\
\text { method }\end{array}$} \\
\hline & & $\not \mathcal{S}$ & $P B$ & $\mathscr{H S}$ & $P B$ \\
\hline $\begin{array}{l}\text { All infarcts } \\
\text { Anterior infarcts } \\
\text { Inferior infarcts }\end{array}$ & $\begin{array}{l}\text { Integral CK } \\
\text { Peak CK } \\
\text { Integral CK } \\
\text { Peak CK } \\
\text { Integral CK } \\
\text { Peak CK }\end{array}$ & $\begin{array}{l}0.68 \\
0.70 \\
0.76 \\
0.84 \\
0.67 \\
0.64\end{array}$ & $\begin{array}{l}0.53 \\
0.55 \\
0.75 \\
0.75 \\
0.53 \\
0.51\end{array}$ & $\begin{array}{l}0.71 \\
0.72 \\
0.76 \\
0.83 \\
0.71 \\
0.68\end{array}$ & $\begin{array}{l}0.64 \\
0.62 \\
0.72 \\
0.68 \\
0.66 \\
0.63\end{array}$ \\
\hline
\end{tabular}




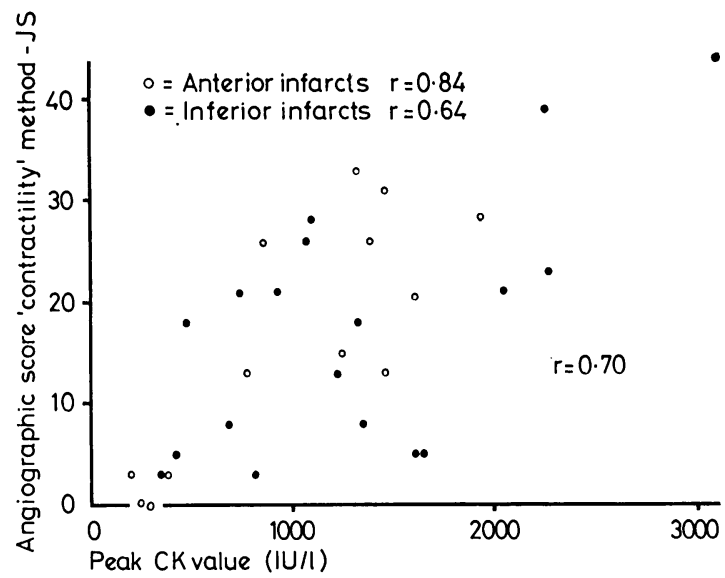

Fig. 5 Comparison of myocardial infarct size assessed by peak $C K$ value and angiocardiography.

Three patients with ischaemic myocardium evidenced by angina on stress testing and five who were on beta-adrenergic blocking drugs at the time of angiography did not appear to have a different ventriculographic "score" relative to $\mathrm{CK}$, and their exclusion from the series would not have significantly affected the relations.

\section{Discussion}

A major problem in the evaluation of therapeutic interventions in myocardial infarction is the difficulty in assessing accurately their effects on myocardial infarct size. Of the methods which are available, analysis of serum $\mathrm{CK}$ is a widely used method of assessing myocardial damage. ${ }^{256} \mathrm{CK}$ appearance in the serum bears a close relation to myocardial CK depletion ${ }^{9} 10$ which itself has been shown in experimental infarction to correlate well with both myocardial blood flow reduction, ${ }^{19}$ and histological infarct size. ${ }^{1020}$ A good correlation between myocardial damage measured enzymatically and that assessed clinically ${ }^{15}$ and at necropsy ${ }^{7}$ has subsequently been reported, while a recent study ${ }^{8}$ comparing cumulative cardiospecific $\mathrm{CK}$ MB release and two ventriculographic indices of myocardial damage (ejection fraction, and "percentage abnormally contracting segment") has added support to these observations.

The current methods and assumptions in the calculation of $\mathrm{CK}$ appearance, however, have recently been questioned on two major grounds. First, the assumed mono-experimental decay rate and identity of the constant $\left(\mathrm{K}_{\mathrm{D}}\right)$ required for the calculation of integral $\mathrm{CK}$ has been challenged theoretically ${ }^{12}$ and experimentally. ${ }^{21}$ Secondly, in demonstrating in experimental animals that the serum entry ratio may vary with infarct size, Cairns and colleagues have raised the possibility that large infarcts may be underestimated relative to smaller ones. ${ }^{22}$

Our study aimed to provide information on these two issues by comparing the extent of enzyme appearance with the size of the myocardial scar as seen by ventriculography one month later. We have compared angiocardiographic assessments of myocardial damage both with cumulative CK release (which depends on estimates of the rate of removal of $\mathrm{CK}$ from the serum, $\mathrm{K}_{\mathrm{D}}$ ) and the peak $\mathrm{CK}$ value which does not require any such estimate. In addition, as our study contains infarcts of a wide range of severity, the variability of CK release relative to angiocardiographic infarct size can be examined.

Correlation between the two enzymic indices (peak CK and integral CK) was very close $(\mathbf{r}=0.97)$. This is at first sight surprising, because integral CK varies directly with $K_{D}$ which itself varies widely from patient to patient. A mean value for $\mathrm{K}_{\mathrm{D}}$ calculated from previously reported groups of patients had a standard deviation on one-third to one-half of the mean value. ${ }^{15}$ In the present group $(n=32)$, the mean value for $K_{D}$ was 0.00090 $\pm 0.00021 \mathrm{SD}$ ) $\mathrm{min}^{-1}$ so that the standard deviation was approximately 23 per cent of the mean value. Inspection of Fig. 2 shows that a prediction of integral $\mathrm{CK}$ from any value for peak $\mathrm{CK}$ would have a likely error $( \pm 2$ SD about the predicted value) of less than 50 per cent. Thus the departure from a perfect correlation between peak $\mathrm{CK}$ and integral CK is fully accounted for by the range of $\mathrm{K}_{\mathrm{D}}$; curves of $\mathrm{CK}$ appearance in the serum after infarction in other words vary only by their peak values and the exponential slopes of their decay rates from which $K_{D}$ is calculated. This is not surprising since it is know that $\mathrm{CK}$ appearance curves from uncomplicated infarcts have a stereotyped log-normal pattern. ${ }^{23}$ The close correlation of peak CK with integral CK suggests that peak $\mathrm{CK}$ could be used as a more convenient index of infarct size than the more laborious integral method which entails four-hourly blood sampling for 72 hours or longer for calculation of $\mathrm{K}_{\mathrm{D}}$. In two therapeutic trials of propranolol ${ }^{6}{ }^{24}$ we have found comparisons of peak $\mathrm{CK}$ values between treated and control patients to give essentially similar information as comparison of the integrals. Moreover, Armstrong et al..$^{25}$ have argued on theoretical grounds that peak CK levels are closely related to total $\mathrm{CK}$ appearance.

Our radiological technique included biplane films in right anterior oblique and left anterior oblique with cranial axial angulation as these two 


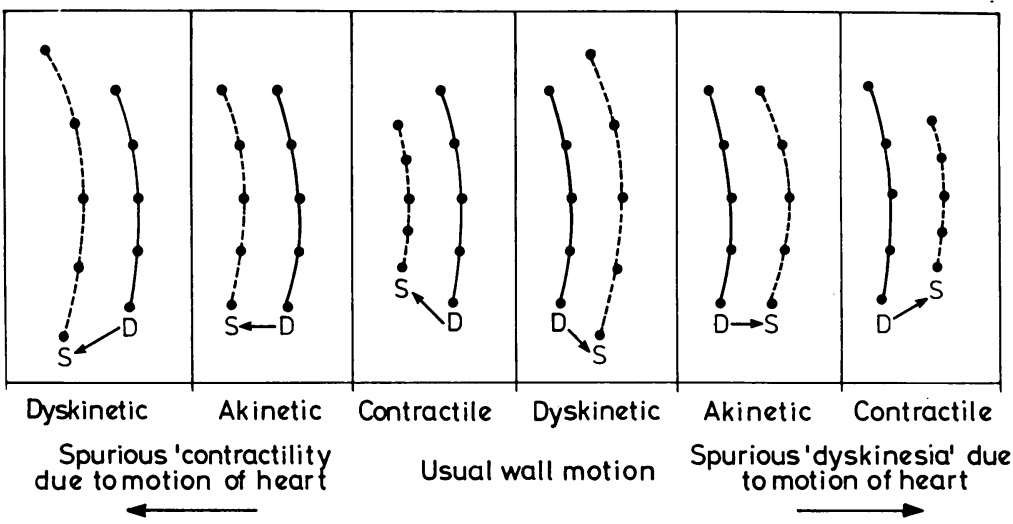

Fig. 6 Diagrammatic representation of pitfalls in the assessment of the contractile state of the ventricular wall by superimposition of diastolic and systolic outlines using external frames of reference. In each diagram the same portion of the ventricular cavity margin is represented in isolation, showing change of position and dimension in diastole (D, continuous line) and systole ( $S$, interrupted line). The diagrammatic individual adjacent portions are separated by dots to emphasise change in dimension. In the centre are the usual appearances of contractility and dyskinesia without interference by motion of the heart as a whole. To each side are examples of possible confusion caused by cardiac motion. The contractile state must be assessed independently of motion.

projections allow visualisation of all segments of myocardium with least foreshortening of the long axis of the left ventricle. As the septal profile represents nearly half the ventricular muscle ${ }^{17}$ this has been taken into account in calculating the "contractility" score.

The analysis of the contractile state of the left ventricular myocardium in our study was done by subjective observation which should allow an experienced analyst to distinguish between changes caused by cardiac motion and variations in the intrinsic contractile behaviour of the region observed. Such subjective analysis must be based on comparison of the relative behaviour of small adjacent components of the profiled area under consideration (Fig. 6). Subjective analysis is frequently difficult and likely to be less reproducible than objective measurements. ${ }^{28}$ It is therefore not surprising that the interobserver correlations were below 0.9 . This is not to say that an objective method necessarily is more accurate in categorising the contractile state of the myocardium. Objective methods using internal frames of reference ${ }^{26-28}$ may be useful but we have not yet attempted to apply them to the assessment of infarct size.

The method described by Rogers et al. ${ }^{8}$ uses an external frame of reference and was applied originally to serial large film studies. It is based on superimposition of systolic and diastolic outlines without allowing for the possibility that a noncontractile region can be moved centrally by adjacent contractile regions or that unusual cardiac motion can cause superimposition or even paradoxical overlap of a contractile region (Fig. 6). It should be noted that the definitions of "akinesia", "dyskinesia", and "hypokinesia" used by Rogers et al. ${ }^{8}$ did not relate directly to the contractile behaviour of the muscle, and their conclusions regarding lack of correlation between "hypokinesia" and infarction do not apply to more accurate assessments of the contractile state. Thus nine $(28 \%)$ of our patients with small infarcts showed only hypokinesia by our method. In the left anterior oblique projection the forward systolic motion of the aortic root and the base of the heart gave an "abnormally contracting segment"8 by superimposition of systolic and diastolic outlines using external frames of reference in 30 patients ( $94 \%$ ) (Fig. 7). In only six cases was this considered to be associated with infarction in the basal septum. In the others it was a result of normal forward motion of the base of the heart. Making allowance for this region, 14 (44\%) of our patients would have shown no abnormally contracting segment by superimposition of systolic on diastolic outlines using an external frame of reference (Fig. 7).

This evaluation of myocardial damage by left ventriculography makes the basic assumption that the severity of infarction in a given area is reflected by the severity of subsequent contractile disorder. A small subendocardial infarct should show only slight hypokinesia or normal appearances, more severe infarction should result in more severe hypokinesia or akinesia, depending on the propor- 
Fig. 7 Superimposed end-diastolic (continuous lines) and end-systolic (interrupted lines) left ventricular outlines in right anterior oblique (RAO) and left anterior oblique with cranial axial angulation (LAO) projections in three patients. External frame of reference determines the positions of the tracings as the $\mathrm{x}$-ray beam and patient were not moving and there was no respiratory motion, the frames being traced on to the same piece of paper in the analysing projector. These examples were selected to illustrate some of the difficulties in applying this method to the assessment of contractility. All three cases in LAO show an "abnormally contracting segment" of the basal septal region due to cardiac motion. Only in (b) was this associated with hypokinesia. In (a) the infarcted area was readily recognised subjectively but would have escaped detection by this method. In (b) where a larger infarct associated with an akinetic area inferiorly caused a severe irregularity of contour, cardiac motion, nevertheless, allowed the whole systolic contour to fall within the diastolic contour. In (c) the hypokinetic anterolateral contour in $R A O$ is made by cardiac motion to appear more contractile than the normal inferior surface. Numbers indicate the subjective assessment of the contour margins as in Fig. 2.

tion of the contractile muscle and scar tissue, and dyskinesia should indicate severe infarction with thinning of the ventricular wall. These assumptions are not always correct as the contractile behaviour can be modified by ischaemia or drugs. ${ }^{27}$ Moreover, though good wall motion means normal myocardium, normal myocardium does not necessarily mean good wall motion. ${ }^{29}$ Though our patients taking beta-blocking drugs and those who had angina on exercise did not show a different correlation from the other patients, it is possible that the ventriculographic appearances overestimated myocardial necrosis to a variable degree by showing contraction abnormalities in normal myocardium adjacent to the infarct or in viable myocardium perfused by a stenotic vessel. ${ }^{29}$ Other sources of error include (in the case of left ventriculography) technical failure to visualise all left ventricular myocardial segments, the difficulty of analysing the various contractile states, and inability to assess right ventricular abnormalities which are common in inferior infarction. ${ }^{30}$ Our results suggest that associated right ventricular infarction in some cases
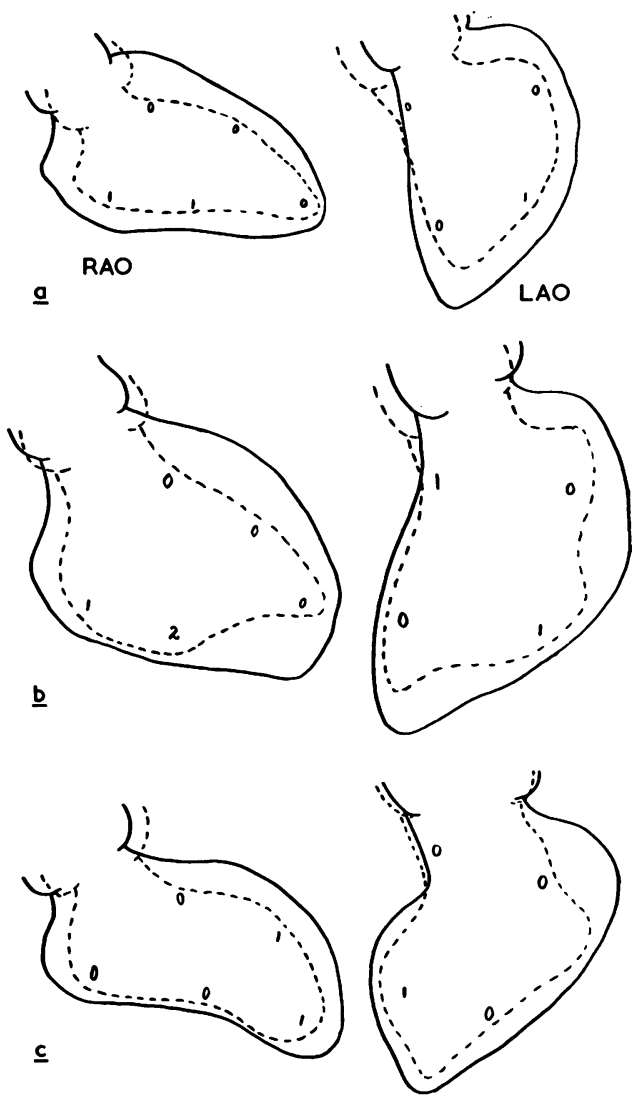

of inferior myocardial infarction could explain both their poorer correlation with angiographic indices and their tendency for higher enzyme levels for a given degree of left ventricular impairment.

Sources of error in the enzyme measurements include biological variation in the $\mathrm{CK}$ content of normal myocardium and its depletion by infarction, ${ }^{11}$ contribution of non-cardiac CK to total CK appearance, and variations in serum entry ratio probably caused by varying degrees of CK degradation locally within the infarct. ${ }^{22}$ For reasons discussed earlier, it is possible that variability of $K_{D}$ may be of somewhat lesser importance.

Considering all of these possible sources of error, it is not surprising that the correlations we have shown between enzymic and cineangiocardiographic indices are less than ideal, varying considerably with the method used for quantification of the angiocardiograms as well as with the differing subjective impressions of different radiologists who interpret them. Moreover, the invasive nature of ventriculography and its restriction to patients without previous myocardial damage makes it 
unsuitable for routine use. At the present time there is no "gold standard" for the measurement of infarct size, and its severity is perhaps best assessed by the combined use of clinical, enzymic, and electrocardiographic indices. To these methods can be added others such as ventriculography, nuclear imaging, or echocardiography, where these are specifically indicated. Of laboratory-based methods which are available, the enzymic method is the most reliable. Peak CK activity based on fourhourly blood sampling may provide sufficient information as an index of myocardial infarct size for an end-point for the clinical trial of therapeutic agents which are designed to reduce it.

We are grateful to Dr E A Harris for assistance with statistical analysis.

\section{References}

'Sobel BE, Breshahan GF, Shell WE, Yoder RD. Estimation of infarct size in man and its relation to prognosis. Circulation 1972; 46: 640-8.

${ }^{2}$ Shell WE, Sobel BE. Protection of jeopardized ischemic myocardium by reduction of ventricular afterload. $N$ Engl f Med 1974; 291 : 481-6.

${ }^{3}$ Maroko PR, Hillis LD, Muller JE, et al. Favorable effects of hyaluronidase on electrocardiographic evidence of necrosis in patients with acute myocardial infarction. N Engl f Med 1977; 296: 898-903.

${ }^{4}$ Heng MK, Norris RM, Singh BN, Barratt-Boyes C. Effects of glucose and glucose-insulin-potassium on haemodynamics and enzyme release after acute myocardial infarction. Br Heart $\mathcal{f}$ 1977; 39: 748-57.

${ }^{5}$ Gowda SK, Gillespie TA, Byrne JD, Ambos HD, Sobel B, Roberts R. Effects of external counterpulsation on enzymatically estimated infarct size and ventricular arrythmia. $B r$ Heart $\mathcal{f}$ 1978; 40: 308-14.

'Peter T, Norris RM, Clarke ED, et al. Reduction of enzyme levels by propranolol after acute myocardial infarction. Circulation 1978; 57: 1091-5.

'Bleifeld W, Mathey D, Hanrath P, Buss H, Effert S. Infarct size estimated from serum creatine phosphokinase in relation to left ventricular hemodynamics. Circulation 1977; 55: 303-11.

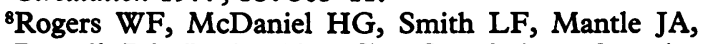
Russell RA Jr, Rackley CE. Correlation of angiographic estimates of myocardial infarct size and accumulated release of creatine kinase $M B$ isoenzyme in man. Circulation 1977; 56: 199-205.

'Shell WE, Kjekshus JK, Sobel BE. Quantitative assessment of the extent of myocardial infarction in the conscious dog by means of analysis of serial changes in serum creatine phosphokinase activity. $f$ Clin Invest 1971; 50: 2614-25.

${ }^{10}$ Vatner SK, Baig H, Manders WT, Maroko PR. Effects of coronary artery reperfusion on myocardial infarct size calculated from creatine kinase. $\mathcal{F}$ Clin Invest 1978; 61 : 1048-56.

${ }^{11}$ Roe CR, Starmer CF. A sensitivity analysis of enzymic estimation of infarct size. Circulation 1975; 52: 1-5.

${ }^{12}$ Slutsky AS. Individualized values for the disappearance rate parameter $\left(K_{d}\right)$ in the enzymatic estimation of infarct size. Circulation 1977; 56: 545-7.

${ }^{13}$ Roe CR, Cobb FR, Starmer CF. The relationship between enzymatic and histologic estimates of the extent of myocardial infarction in conscious dogs with permanent coronary occlusion. Circulation 1977; 55: 438-49.

${ }^{14}$ Rosalki SB. An improved procedure for serum creatine phosphokinase determination. f Lab Clin Med 1967; 69: 696-705.

${ }^{15}$ Norris RM, Whitlock RML, Barratt-Boyes C, Small $\mathrm{CW}$. Clinical measurements of myocardial infarct size. Modification of a method for the estimation of total creatine phosphokinase release after myocardial infarction. Circulation 1975; 51: 614-20.

${ }^{16} \mathrm{Judkins}$ MD. Selective coronary cinearteriography. Part I: A percutaneous transfemoral technic. Radiology 1967; 89: 815-24.

${ }^{17}$ Reiner L, Mazzoleni A, Rodriguez FL, Freudenthal RR. Weight of the human heart. Arch Pathol 1959; 68: $58-73$.

${ }^{18}$ Brandt PWT, Partridge JB, Wattie WJ. Coronary arteriography; method of presentation of the arteriogram report and a scoring system. Clin Radiol 1977; 28: $361-365$.

${ }^{19}$ Heng MK, Singh BN, Norris RM, John MB, Elliot R. Relationship between epicardial ST segment elevation and myocardial ischemic damage after experimental coronary artery occlusion in dogs. 7 Clin Invest 1976; 58: 1317-26.

${ }^{20} \mathrm{Kjekhus} \mathrm{JK}$, Sobel BE. Depressed myocardial creatine phosphokinase activity following experimental myocardial infarction in rabbit. Circ Res 1970; 27: 403-14.

${ }^{21}$ Sobel BE, Markham J, Karlsberg RP, Roberts R. The nature and disappearance of creatine kinase from the circulation and its influence on enzymatic estimation of infarct size. Circ Res 1977; 41: 836-44.

${ }^{22}$ Cairns JA, Missirlis E, Fallen EL. Infarct size from serial CPK: variability of serum entry ratio with infarct size and model (abstract). Circulation 1977; 55 \& 56, suppl III : 70.

${ }^{23}$ Shell WE, Lavell JF, Covelle JW, Sobel BE. Early estimation of myocardial damage in conscious dogs and patients with evolving acute myocardial infarction. f Clin Invest 1973; 52: 2579-90.

${ }^{24}$ Norris RM, Clarke ED, Sammel NL, Smith WM, Williams B. Protective effect of propranolol in threatened myocardial infarction. Lancet 1978; 2: 907-9.

${ }^{25}$ Armstrong PW, Watts DG, Hamilton DC, Chiong MA, Parker JO. Template model for serial creatine kinase analysis in myocardial infarction. Circulation 1977; 55 \& 56, suppl III: 20 .

${ }^{28}$ Chaitman BR, DeMots H, Bristow JD, Rösch J, Rahimtoola SH. Objective and subjective analysis of left ventricular angiograms. Circulation 1975; 52: 420-5.

${ }^{27}$ Herman MV, Heinle AH, Klein MD, Gorlin R. Localized disorders in myocardial contraction. Asynergy and its role in congestive heart failure. $N$ Engl f Med 1967; 277: 222-32. 
${ }^{28}$ Ideker RE, Behar VS, Wagner GS, et al. Evaluation of asynergy as an indicator of myocardial fibrosis. Circulation 1978; 57: 715-25.

${ }^{29}$ Hutchings GM, Bulkley BN, Ridolfi RL, Griffith LSC, Lohr FT, Piasio MA. Correlation of coronary arteriograms and left ventriculograms with post mortem studies. Circulation 1977; 56: 32-7.
${ }^{30}$ Sharpe DN, Botvinick EH, Shames DM, et al The noninvasive diagnosis of right ventricular infarction. Circulation 1978; 57: 483-90.

Requests for reprints to Dr N L Sammel, St Vincent's Hospital, Darlinghurst, NSW 2010, Australia. 